Revista Brasileira de Meteorologia, v.26, n.2, 313 - 321, 2011

\title{
AVALIAÇÃO DA RELAÇÃO SECA/PRODUTIVIDADE AGRÍCOLA EM CENÁRIO DE MUDANÇAS CLIMÁTICAS
}

\author{
ROZIANE SOBREIRA DOS SANTOS ${ }^{1,2}$, LUIZ CLÁUDIO COSTA ${ }^{2}$, GILBERTO CHOHAKU \\ SEDIYAMA $^{2}$, BRAULIRO GONÇALVES LEAL ${ }^{3}$, ROBSON ALVES DE OLIVEIRA ${ }^{2}$ E FLÁVIO \\ BARBOSA JUSTINO ${ }^{2}$
}

\author{
${ }^{1}$ Universidade Federal de Rondônia (UNIR), Departamento de Matemática e Estatística, Ji-Paraná, MG, Brasil \\ ${ }^{2}$ Universidade Federal de Viçosa (UFV), Departamento de Engenharia Agrícola, Viçosa, MG, Brasil \\ ${ }^{3}$ Universidade Federal do Vale do São Francisco (UNIVASF), Juazeiro, BA, Brasil \\ roziane.santos@ufv.br,1.costa@ufv.br,g.sediyama@ufv.br, brauliro@gmail.com, \\ robson.oliveira@ufv.br, fjustino@ufv.br
}

Recebido Junho 2009 - Aceito Agosto 2010

\begin{abstract}
RESUMO
As mudanças climáticas alertam para um possível aumento de eventos meteorológicos extremos em todo o mundo, sendo crescente a preocupação de como o clima pode mudar o ambiente e afetar a produção das culturas agrícolas. Este estudo investiga a relação entre a produtividade agrícola e a seca em algumas mesorregiões do estado de Minas Gerais, em cenários de mudanças climáticas. Foram utilizados dados meteorológicos diários projetados pelo modelo ECHAM5/MPI-OM, para o período de 2008 a 2020 para o cenário A1B. Utilizou-se a metodologia da zona agroecológica (AEZ) para estimar a produtividade futura do milho. Empregou-se o índice de seca $Z$ de Palmer em um modelo de regressão linear com a produtividade do milho estimada pela metodologia da AEZ. O desempenho dos modelos foi verificado por meio das estatísticas: coeficiente de determinação $\left(\mathrm{r}^{2}\right)$, raiz do erro quadrático médio (RMSE), erro absoluto médio (MAE) e índice de concordância de Willmott (d). Os resultados do índice de concordância de Willmott variaram entre 0,48 e 0,90 , e os valores de $r^{2}$ foram pouco expressivos. Contudo, a produtividade estimada pela metodologia AEZ projetou maiores perdas na produtividade do milho devido a limitações por água para os anos agrícolas de 2008/2009, 2009/2010, 2014/2015, 2018/2019 para as mesorregiões Triângulo/Alto Paranaíba, Central Mineira e Jequitinhonha.
\end{abstract}

Palavras-chave: Cenário A1B, Seca agrícola, Zona agroecológica

\begin{abstract}
EVALUATION OF AN AGRICULTURAL DROUGHT/YIELD RELATIONSHIP IN A SCENARIO OF CLIMATE CHANGE

This study investigates the relationship between crop yields and drought in the state of Minas Gerais for of climate change scenarios. Climate changes have warned due to possible increases in meteorological extremes worldwide and the uncertainties on how the climatic events might change the environment and affect world agricultural production in the future. The ECHAM5/MPI-OM model daily weather data projected for the 2008 to 2020 period, based on the A1B scenario, was used. The methodology of the agroecological zone (AEZ) to estimate the future maize yield was used. The index of the Z Palmer drought and the estimated productivity by AEZ methodology were compared by a linear correlation model. The performance of the models was verified by the statistic parameters: coefficient of determination $\left(\mathrm{r}^{2}\right)$, the root mean square error (RMSE), mean absolute error (MAE) and Willmott agreement index (d). The results of the Willmott agreement index ranged from 0.48 to 0.90 and $r^{2}$ presented low values. However, the productivity estimated by AEZ methodology projected greater maize yield losses due to water supplies limitations for the agricultural years of 2008/2009, 2009/2010, 2014/2015 and 2018/2019 for the mesoregions of Triângulo/Alto Paranaíba, Central Mineira and Jequitinhonha.
\end{abstract}

Keywords: A1B Scenario, agricultural drought, agro-ecological zones. 


\section{INTRODUÇÃO}

Analisar os impactos das mudanças climáticas com a finalidade de compreender o que pode ser feito para fins de mitigação e, ou, adaptação a essas mudanças nas diversas atividades humanas, tem sido uma grande preocupação de pesquisadores e governos em todo o mundo. Com isso, muitos estudos estão direcionados para essa questão. Em destaque vêm os relatórios do Painel Intergovernamental de Mudanças Climáticas (IPCC), base de pesquisa para muitos dos grupos de pesquisadores pelo mundo. Em seus últimos relatórios, o IPCC (2007) destaca que as causas do aquecimento global são, principalmente, devido às emissões antropogênicas de gases de efeito estufa (IPCC, 2007).

Quando se analisa os possíveis impactos das mudanças climáticas, os relatórios do IPCC indicam que os países em desenvolvimento são, de modo geral, os mais vulneráveis. No Brasil, há muitos exemplos de impactos adversos da variabilidade natural do clima causadores de enormes prejuízos econômicos e sociais, causadas principalmente pelas secas, inundações e geadas.

A questão do possível aumento dos extremos climáticos, automaticamente, remete ao problema da fragilidade das populações e dos ecossistemas a estas mudanças. Sendo assim, espera-se que, mantidas as condições atuais de desenvolvimento, a vulnerabilidade do Brasil às mudanças climáticas seja muito alta.

De modo geral, todos os setores são vulneráveis às mudanças climáticas. Entretanto, o setor agrícola, devido a sua forte dependência ao clima e a necessidade cada vez maior da produção de alimentos, tem sido objeto de muitos estudos em todo o mundo. Muitos deles vêm mostrando que as condições climáticas futuras poderão ter impactos substanciais na produtividade das culturas (Luo et al., 2005; Richter e Semenov, 2005; Zhang e Liu, 2005; Challinor et al., 2007; Lobell, 2007; Barrios et al., 2008).

Oliveira (2007) projetou quedas na produtividade potencial das culturas de milho e feijão para as mesorregiões de
Minas Gerais, para os anos de 2050 e 2080, quando comparada à produtividade potencial simulada para o ano base de 2000, usando o cenário A2 do modelo HadCM3, sem avaliar o efeito do $\mathrm{CO}_{2}$. Essa queda foi devida, principalmente, às temperaturas mais altas, que diminuem a assimilação de carbono pela cultura, decorrente do encurtamento das fases fenológicas e aumento da taxa de respiração de manutenção. Silva Júnior (2007), simulando a produtividade do milho para os anos de 2020, 2050 e 2080 e utilizando o modelo CERES-MAIZE para os cenários de mudanças climáticas A2 e B2, verificou uma diminuição da produtividade devido ao aumento de temperatura e uma redução no ciclo vegetativo, em ambos cenários, sendo mais significativo para o cenário A2. No entanto, ainda existe uma série de incertezas sobre tais impactos, uma vez que a produtividade das culturas depende de uma série de fatores biofísicos e socioeconômicos, que são difíceis de quantificar (Ewert et al., 2005).

Com o aquecimento global, em um futuro próximo, espera-se um cenário de clima extremo com secas, inundações e ondas de calor mais intensas (Pinto et al., 2003). Burke et al. (2006) projetam uma previsão de aumento de eventos extremos de seca para a segunda metade do século XXI. Hughes e Diaz (2008) verificaram com modelos de clima forçado, que nos próximos 50 anos, as secas poderão ser mais intensas e extensas na América do Norte.

Para um país onde a agricultura tem enorme expressão na economia, há uma deficiência em estudos prospectivos sobre os possíveis impactos das mudanças climáticas na produção agrícola. No Brasil, ainda há poucos estudos realizados sobre o reflexo das mudanças climáticas e seus impactos na agricultura.

A análise dos possíveis impactos das mudanças climáticas na agricultura permite o planejamento de ações e a obtenção de tecnologias necessárias para enfrentar tais mudanças. Nesse contexto, é de extrema relevância analisar como as mudanças climáticas poderão afetar as safras agrícolas. Sendo assim, objetivou-se com este trabalho analisar, em cenários futuros, a influência da seca na produtividade do milho

Tabela 1 - Referências geográficas do ponto central das Mesorregiões e período de estudado

\begin{tabular}{l|c|c|c|c}
\hline \multirow{2}{*}{ Mesorregião (IBGE) } & \multicolumn{3}{c}{${ }^{\mathrm{I}}$ Coordenadas Geográficas: Ponto } & \multicolumn{1}{c}{$\begin{array}{c}\text { Período } \\
\text { Estudado }\end{array}$} \\
\hline Sul/Sudoeste & $19^{\circ} 48^{\prime}(\mathrm{S})$ & $43^{\circ} 48^{\prime}(\mathrm{W})$ & 926 & 2008 a 2020 \\
Jequitinhonha & $16^{\circ} 42^{\prime}(\mathrm{S})$ & $43^{\circ} 54^{\prime}(\mathrm{W})$ & 603 & 2008 a 2020 \\
Vale do Rio Doce & $21^{\circ} 06^{\prime}(\mathrm{S})$ & $44^{\circ} 18(\mathrm{~W})$ & 416 & 2008 a 2020 \\
Campos das Vertentes & $19^{\circ} 12^{\prime}(\mathrm{S})$ & $47^{\circ} 47^{\prime}(\mathrm{W})$ & 943 & 2008 a 2020 \\
Central Mineira & $18^{\circ} 54^{\prime}(\mathrm{S})$ & $42^{\circ} 00^{\prime}(\mathrm{W})$ & 659 & 2008 a 2020 \\
Metropolitana de Belo Horizonte & $20^{\circ} 30^{\prime}(\mathrm{S})$ & $45^{\circ} 36^{\prime}(\mathrm{W})$ & 751 & 2008 a 2020 \\
Triângulo Mineiro/A. Paranaíba & $17^{\circ} 54^{\prime}(\mathrm{S})$ & $41^{\circ} 30^{\prime}(\mathrm{W})$ & 737 & 2008 a 2020 \\
Zona Mata Mineira & $20^{\circ} 48^{\prime}(\mathrm{S})$ & $42^{\circ} 54^{\prime}(\mathrm{W})$ & 536 & 2008 a 2020 \\
\hline
\end{tabular}

${ }^{1}$ Ponto central da célula, ponto em que foi gerada a série de dados do modelo ECMWF que coincide geograficamente com a mesorregião. 
no Estado de Minas Gerais. O foco principal são as projeções de aquecimento global antropogênico propostas nos relatórios do IPCC.

\section{DADOS E METODOLOGIA}

O estudo foi realizado para Estado de Minas Gerais localizado na região Sudeste do país, entre os paralelos $14^{\circ} 13^{\prime}$ $57^{\prime \prime}$ e $22^{\circ} 55^{\prime} 22^{\prime \prime}$ de latitude Sul, e os meridianos de $39^{\circ} 51^{\prime} 23^{\prime \prime}$ e $51^{\circ} 02^{\prime} 45^{\prime \prime}$ a oeste de Greenwich. Considerou-se a divisão do Estado de Minas Gerais em mesorregiões geográficas, conforme critério do Instituto Brasileiro de Geografia e Estatística (IBGE). A Tabela 1 apresenta a localização geográfica e a altitude dos pontos centrais das mesorregiões estudadas e o período de análises.

Foram utilizadas as condições climáticas projetadas pelo modelo de circulação geral da atmosfera ECHAM5/MPIOM, desenvolvido no Max-Planck-Institute for Meteorology em Hamburgo, Alemanha, no período de 2008 a 2020 para o cenário A1B. O modelo ECHAM5/MPI-OM é composto de duas componentes: ECHAM5 para a atmosfera e MPI-OM para o oceano. Detalhes técnicos do desenvolvimento dessas componentes são descritos por Roeckner et al. (2003) para o componente atmosférico e por Marsland et al. (2003) para o modelo oceânico. Esse modelo apresenta resolução aproximadamente de $1,8^{\circ}$ de latitude e de longitude, o que representa uma resolução espacial de aproximadamente 180 x $180 \mathrm{Km}$, na faixa equatorial, com 31 níveis verticais. Os dados foram simulados considerando os pontos centrais das mesorregiões estudadas (Tabela 1).

Para as projeções futuras, foi calculado o índice de anomalia de umidade, índice Z, desenvolvido por Palmer (1965). Este índice considera que o total de precipitação exigida, para manter uma área sob condições econômicas estáveis, depende da média histórica dos elementos meteorológicos e das condições hídricas dos meses precedentes e do mês considerado, baseado nos princípios do balanço entre o suprimento e a demanda de água no solo. O solo é dividido em duas camadas, uma superior (Ss) com capacidade de armazenamento de $25,4 \mathrm{~mm}$ e outra sub-superficial ( $\mathrm{Su})$.

$\mathrm{O}$ índice $\mathrm{Z}$ representa um termo intermediário no cálculo do índice de severidade de seca de Palmer - PDSI (Palmer, 1965). Ele é uma medida das anomalias de umidade mensal e reflete o desvio das condições de umidade, em um determinado mês das condições normais de umidade (Heim Junior, 2002; Keyantash e Dracup, 2002).

Conforme Karl (1986) e Quiring e Papakryiakou (2003), o índice $\mathrm{Z}$ reflete as condições hídricas do mês em questão, sem a influência de meses precedentes, podendo indicar um mês úmido em meio a uma seca prolongada. Sendo assim, é mais indicado aos interesses agrícolas do que o próprio PDSI. Valores negativos do índice $\mathrm{Z}$ denotam períodos secos, enquanto valores positivos ,períodos úmidos:

$$
\mathrm{Z}=\mathrm{d}^{\prime} \times \mathrm{K}
$$

em que, d' é a anomalia hídrica que contabiliza o excesso ou deficiência da precipitação observada $(\mathrm{P})$ em relação à precipitação Climaticamente Apropriada às Condições Existentes $(\hat{\mathrm{P}})$. A anomalia hídrica é dada por:

$$
\begin{aligned}
& \mathrm{d}^{\prime}=\mathrm{P}-\hat{\mathrm{P}} \\
& \hat{\mathrm{P}}=\hat{\mathrm{ET}}+\hat{\mathrm{R}}+\hat{\mathrm{RO}}-\hat{\mathrm{L}} \\
& \hat{\mathrm{ET}}=\alpha \times \mathrm{ETo} \\
& \hat{\mathrm{R}}=\beta \times \mathrm{PR} \\
& \hat{\mathrm{RO}}=\gamma \times \mathrm{PRO} \\
& \hat{\mathrm{L}}=\delta \times \mathrm{PL}
\end{aligned}
$$

Os parâmetros $\alpha, \beta, \gamma$ e $\delta$ são definidos por Palmer (1965) para calcular os valores climaticamente apropriados às condições existentes de evapotranspiração (ET), de recarga $(\hat{\mathrm{R}})$, de escoamento ( $\hat{\mathrm{RO}})$, de perda $(\hat{\mathrm{L}})$ e de precipitação $(\hat{\mathrm{P}})$.

A caracterização climática $(\mathrm{K})$ é dada por:

$$
\mathrm{K}=\frac{17,67}{\sum_{1}^{12} \overline{\mathrm{D}} \mathrm{K}^{\prime}} \mathrm{K}^{\prime}
$$

em que,

$$
\mathrm{K}^{\prime}=1,5 \times \log _{10}\left[\frac{\left(\frac{\overline{\mathrm{ETo}}+\overline{\mathrm{R}}+\overline{\mathrm{RO}}}{\overline{\mathrm{P}}+\overline{\mathrm{L}}}+2,80\right)}{\overline{\mathrm{D}}}\right]+0,50
$$

onde $\bar{D}=$ Média mensal dos valores absolutos de d'.

Para estimar a produtividade potencial do milho, limitada por água para as projeções climáticas futuras, foi utilizada a metodologia da zona agroecológica-AEZ (FAO, 1978; Fischer et al., 2000).

Essa metodologia assume um armazenamento não diferenciado de água no solo de uma camada e calcula o conteúdo 
mensal de umidade com relação à evapotranspiração da planta, a precipitação e resíduo de umidade do mês precedente.

A produtividade potencial da cultura, calculada pela metodologia AEZ, reflete o regime da temperatura e da radiação solar do local em estudo. A metodologia é basicamente ecofisiológica (Kassam, 1977; Fischer et al., 2002) e a aplicação é feita por meio de parâmetros, que dependem da cultura, duração do ciclo de crescimento, índice de área foliar, índice de colheita, produção bruta de matéria seca e do clima (temperatura e radiação). Diversos autores têm utilizado a metodologia AEZ para estimar a produtividade potencial de várias culturas (Deng et al., 2006).

A produtividade potencial (Ymp) da cultura representa o nível de referência de produtividade atingível com alto padrão de manejo da cultura e água, em que não há limitações de clima, água e nutrientes e sem ataque de pragas e doenças (Doorenbos e Kassam, 1979):

$$
\begin{aligned}
& \mathrm{Ymp}=\mathrm{cL} \times \mathrm{cN} \times \mathrm{cH} \times \mathrm{G} \times[\mathrm{F}(\mathrm{a}+\mathrm{b} \times \mathrm{ym}) \\
& \mathrm{F}=\frac{(\text { Rso- } 0,5 \times \mathrm{Rs})}{(0,8 \times \text { Rso })}
\end{aligned}
$$$$
\text { yon }=31,653+0,5477 \times \text { Rso }
$$

$$
y c=104,66+0,9061 \times \text { Rso }
$$

onde $\mathrm{Ymp}=$ produtividade potencial $\left(\mathrm{kg} \mathrm{ha}^{-1}\right), \mathrm{cL}=$ correção em função do desenvolvimento da cultura e área foliar (adimensional), $\mathrm{cN}=$ correção para a produção de matéria seca (adimensional), $\mathrm{cH}=$ índice de colheita (adimensional), $\mathrm{G}=$ duração do ciclo de crescimento (dias), $\mathrm{F}=$ fração do dia em que o sol fica encoberto por nuvens (adimensional), Rso = radiação solar na superfície para dias claros $\left(\mathrm{MJ} \mathrm{m}^{-2} \mathrm{~d}^{-1}\right), \mathrm{Rs}=$ radiação global à superfície $\left(\mathrm{MJ} \mathrm{m}^{-2} \mathrm{~d}^{-1}\right)$, yon $=$ taxa de produção bruta de matéria seca da cultura-padrão em dias completamente nublado $\left(\mathrm{kg} \mathrm{ha}^{-1} \mathrm{~d}^{-1}\right), \mathrm{yc}=$ taxa de produção bruta de matéria seca da cultura-padrão em dias sem nuvens $\left(\mathrm{kg} \mathrm{ha}^{-1} \mathrm{~d}^{-1}\right)$ e $\mathrm{ym}=$ taxa de produção bruta de matéria seca $\left(\mathrm{kg} \mathrm{ha}^{-1} \mathrm{~d}^{-1}\right)$.

Os valores de a, b, c e d são iguais a $0,8,0,01,0,5$ e 0,025 , respectivamente, para ym $\geq 20 \mathrm{~kg} \mathrm{ha}^{-1} \mathrm{~d}^{-1}$, e iguais a $0,5,0,025$, 0 e 0,05 , respectivamente, para ym $<20 \mathrm{~kg} \mathrm{ha}^{-1} \mathrm{~d}^{-1}$.

Os dados de Rso e Rs foram convertidos de $\mathrm{MJ} \mathrm{m}^{-2} \mathrm{~d}^{-1}$ para cal cm${ }^{-2} \mathrm{~d}^{-1}$, para serem utilizados como modelo de produtividade da zona agroecológica (Doorenbos e Kassam, 1979).

As Equações 12 e 13 foram desenvolvidas por Leal (2000), para regiões selecionadas de Minas Gerais.

A produtividade limitada por água é calculada aplicando fatores de redução relacionados ao estádio de crescimento da cultura. Os fatores relacionam a redução relativa na produtividade, expressa como $(1-\mathrm{Ya} / \mathrm{Ymp})$ para o déficit de evapotranspiração (1 - ETr/ETm). Nessa formulação, Ya e Ymp denotam a produtividade limitada por água e potencial, respectivamente. A sigla ETr refere-se a evapotranspiração real da cultura e a sigla ETm a evapotranspiração máxima da cultura. Essa relação é expressa por:

$$
1-\frac{\mathrm{Ya}}{\mathrm{Ymp}}=\mathrm{k}_{\mathrm{y}} \times\left(1-\frac{\mathrm{ETr}^{\mathrm{c}}}{\mathrm{ETm}}\right)
$$

Logo, Ya é dada por:

$$
\mathrm{Ya}=\left(1-\mathrm{k}_{\mathrm{y}} \times\left(1-\frac{E T r^{\mathrm{c}}}{\mathrm{ETm}}\right)\right) \times \mathrm{Ymp}
$$

em que, ky é o coeficiente que expressa a sensibilidade da cultura ao déficit hídrico.

O valor de Ya refere-se a matéria seca, portanto, corrigida para $13 \%$ de umidade, isto é, para umidade de grãos comercializados.

As simulações de produtividade consideram as práticas de manejo e que as cultivares serão, no futuro, as mesmas adotadas atualmente.

Para a data de plantio, considerou-se o início do período chuvoso. O início do período chuvoso foi determinado como a data depois do dia primeiro de outubro, quando a precipitação acumulada em um ou dois dias consecutivos alcança, no mínimo, $20 \mathrm{~mm}$, desde que não haja a ocorrência de um período seco, nos 30 dias seguintes, que exceda dez dias consecutivos. Foi considerado um período seco, os dias com precipitação inferior a $0,5 \mathrm{~mm}$.

Os valores dos parâmetros e coeficientes foram baseados em Doorenbos e Kassam (1979) e Fischer et al. (2002).

Os valores de produtividade limitada por água (Ya) foram ajustados em um modelo de regressão, que considera o índice de seca $(Z)$ como variável independente e Ya como variável dependente. As análises são feitas considerando o índice acumulado durante o ciclo (outubro a janeiro) e o índice de cada mês do ciclo.

O desempenho dos modelos foi verificado por meio das seguintes medidas estatísticas: coeficiente de determinação $\left(\mathrm{r}^{2}\right)$, raiz do erro quadrático médio (RMSE, siga em inglês), o erro absoluto médio (MAE, siga em inglês) e o índice de concordância (d) de Willmott (1982). As medidas estatísticas são dadas pelas seguintes equações:

$$
\mathrm{r}^{2}=\frac{\sum_{\mathrm{i}=1}^{\mathrm{n}}\left(\mathrm{P}_{\mathrm{i}}-\overline{\mathrm{O}_{\mathrm{i}}}\right)^{2}}{\sum_{\mathrm{i}=1}^{\mathrm{n}}\left(\mathrm{O}_{\mathrm{i}}-\overline{\mathrm{O}_{\mathrm{i}}}\right)^{2}}
$$


O coeficiente de determinação $\left(\mathrm{r}^{2}\right)$ mede a proporção da variabilidade da produtividade estimada pela metodologia AEZ, que é explicada pela produtividade estimada pelo índice Z.

O RMSE e MAE são medidas de erros usados para representar as diferenças médias entre os valores estimados pelo índice $\mathrm{Z}(\mathrm{P})$ e os valores estimados pela metodologia da zona agroecológica (O). O RMSE fornece uma informação em relação à dispersão dos dados, ou seja, o grau de espalhamento obtido na comparação com os valores estimados. O MAE é menos sensível a valores extremos. O RMSE e o MAE são calculados, respectivamente, por:

$$
\begin{aligned}
\text { RMSE } & =\sqrt{\frac{\sum_{i=1}^{n}\left(P_{i}-O_{i}\right)^{2}}{n}} \\
\text { MAE } & =\frac{\sum_{i=1}^{n}\left|P_{i}-O_{i}\right|}{n}
\end{aligned}
$$

O índice de concordância de Willmott (d) mede o grau em que os dados estimados pelo índice $Z(P)$ se aproximam dos dados estimados pela metodologia AEZ (O). Este índice varia de zero a um, sendo zero, nenhuma concordância e um, concordância perfeita. O índice de concordância de Willmott é dado por:

$$
\mathrm{d}=1-\frac{\sum_{\mathrm{i}=1}^{\mathrm{n}}\left(\mathrm{P}_{\mathrm{i}}-\mathrm{O}_{\mathrm{i}}\right)^{2}}{\sum_{\mathrm{i}=1}^{\mathrm{n}}\left(\left|\mathrm{P}_{\mathrm{i}}-\overline{\mathrm{O}}_{\mathrm{i}}\right|+\left|\mathrm{O}_{\mathrm{i}}-\overline{\mathrm{O}}_{\mathrm{i}}\right|\right)^{2}}
$$

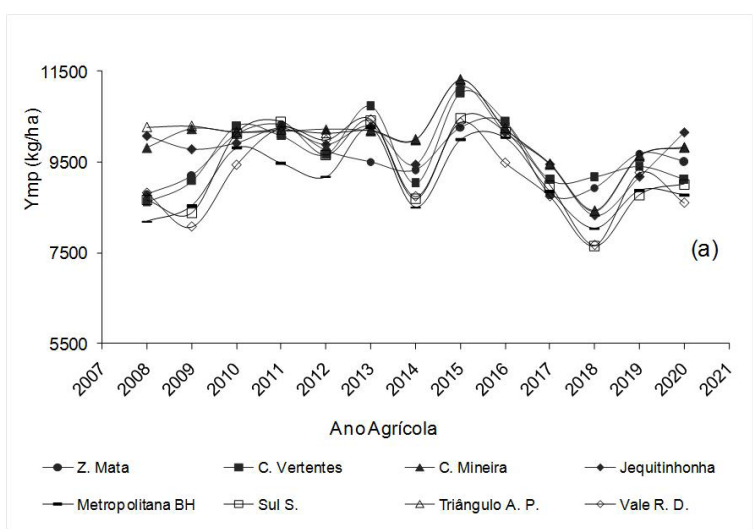

\section{RESULTADOS}

As estimativas da produtividade potencial (Ymp) e da produtividade limitada por água (Ya), para a cultura do milho, em resposta às condições climáticas futuras simuladas pela metodologia AEZ, com os dados diários do cenário A1B do modelo ECHAM5/MPI-OM são apresentadas na Figura 1 (a, b) para cada uma das mesorregiões estudadas. As estimativas de produtividade potencial apresentam um comportamento muito similar entre as mesorregiões. As exceções se dão para as mesorregiões Jequitinhonha, Triângulo/Alto Paranaíba e Central Mineira, que nos anos agrícolas de 2007/2008 e 2008/2009 projetam produtividade potencial superior às demais (Figura 1 a). As variações na produtividade potencial estimada em uma mesma mesorregião são devidas, principalmente, ao aumento e ao decréscimo na temperatura média do ciclo ao longo dos anos e alterações nos valores médios do ciclo da fração do dia em que o sol fica encoberto por nuvens. Ao adicionar a limitação por água na produtividade potencial (Ya), observa-se um comportamento semelhante entre as mesorregiões Triângulo/Alto Paranaíba e Central Mineira, com estimativas de quedas significantes nos anos agrícolas de 2008/2009, 2009/2010 e 2018/2019. Na mesorregião Zona da Mata, a maior limitação por água é estimada para o ano agrícola de 2010/2011. As demais mesorregiões apresentam um comportamento muito similar entre si, em relação aos anos agrícolas que apresentam alguma deficiência hídrica (Figura 1 b). As estimativas apontam que a mesorregião Campo das Vertentes será pouco afetada pelo déficit hídrico.

A Figura 2 apresenta as estimativas de perdas na produtividade estimada pela metodologia da zona agroecológica devido a limitações por água para as mesorregiões. Em todas as localidades, as estimativas apontam para perdas nos anos

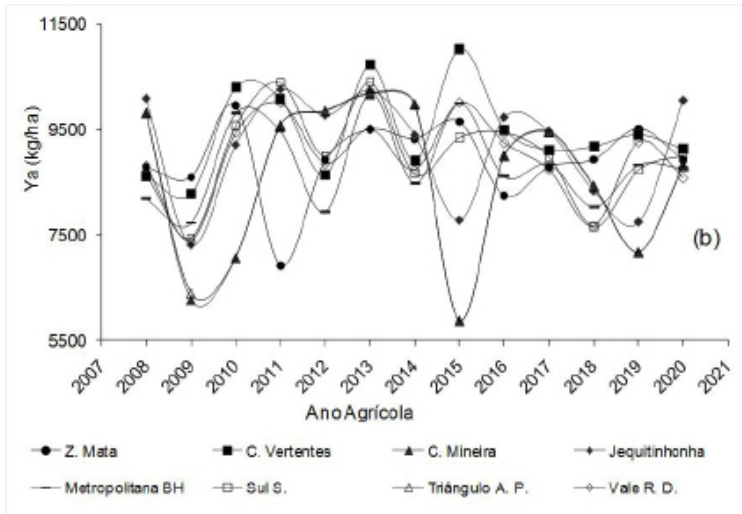

Figura 1 - (a) - Variação das estimativas da produtividade potencial (Ymp); (b) Variação das estimativas de produtividade limitada por água (Ya) do milho simuladas a partir dos dados diários para o cenário A1B do modelo ECHAM5/MPI-OM no período de 2008 a 2020, para as mesorregiões apresentadas na legenda 
agrícolas de 2008/2009, 2011/2012 e 2015/2016, devido à deficiência hídrica. As maiores perdas são estimadas para as mesorregiões Triângulo/Alto Paranaíba, Central Mineira e Jequitinhonha na safra de 2014/2015. Essas também são as mesorregiões mais afetadas pela deficiência hídrica. Para a mesorregião Zona da Mata estima-se uma perda significante para o ano agrícola de 2010/2011. Regiões como Jequitinhonha, que atualmente sofrem perdas agrícolas devido aos baixos índices de precipitação, tendem a continuar com problemas de déficit hídrico na próxima década (Figura 2). Ainda, considerando o cenário A1B do modelo ECHAM5/MPI-OM, é projetada uma tendência de déficit hídrico para a mesorregião Triângulo/Alto Paranaíba, umas das maiores produtoras de milho no momento e também, para a mesorregião Central Mineira. Na mesorregião Zona da Mata, apenas em dois anos agrícolas são estimadas as perdas na produtividade do milho devido ao déficit hídrico (Figura 2). Entretanto, como as demais localidades apresentam uma tendência, que de certa forma se mantém ao longo da década, essa queda de precipitação nos anos agrícolas de 2010/2011 e 2015/2016 pode ser atribuída a alguma deficiência do modelo. Demonstrando, que nesta região, não há estimativas de perdas expressivas. Para as demais regiões estudadas, considerando o cenário A1B do modelo ECHAM5/MPI-OM, não se projetam perdas devido à deficiência hídrica (Figura 2). Portanto, caso as projeções do cenário A1B do modelo ECHAM5/MPI-OM se confirmem, serão necessários investimentos em implantação e, ou, aprimoramento de sistemas de irrigação nas mesorregiões Jequitinhonha, Triângulo/Alto Paranaíba e Central Mineira.

Os resultados do desempenho do índice $\mathrm{Z}$ comparado com a metodologia da zona agroecológica para as mesorregiões estudadas em Minas Gerais são apresentados na Tabela 2 e Figura 3. A Figura 3 apresenta a variação na produtividade estimada pelo índice $Z$ para os meses em que se obteve o melhor

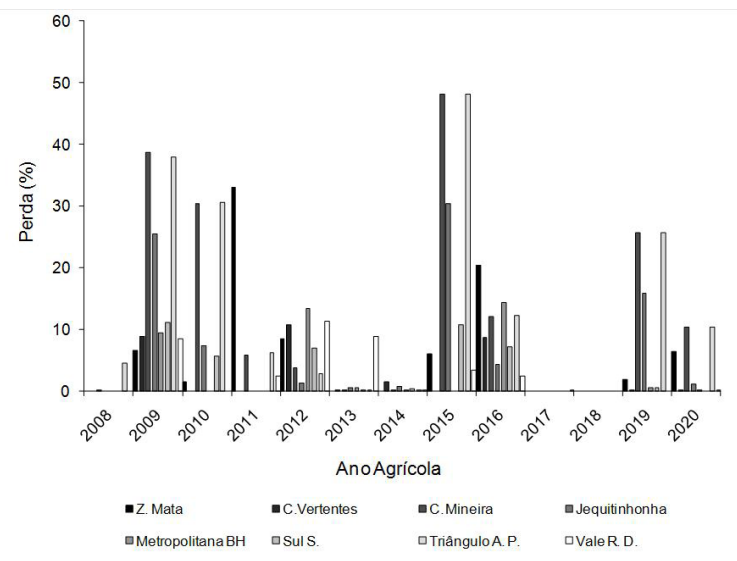

Figura 2 - Estimativas de perdas por limitação hídrica na produtividade do milho simulada a partir de dados diários para o cenário A1B do modelo ECHAM5/MPI-OM no o período de 2008 a 2020, para as mesorregiões apresentadas na legenda ajuste com a produtividade estimada pela zona agroecológica. $\mathrm{O}$ índice Z, calculado com os dados do cenário A1B do modelo ECHAM5/MPI-OM para o mês de dezembro, apresentou os melhores ajustes para as estimativas de produtividade, exceto para a mesorregião Sul e Sudoeste (Tabela 2), para a qual se destacou o ajuste para todo o ciclo (índice $\mathrm{Z}$ acumulado nos meses de outubro a janeiro), e a mesorregião Vale do Rio Doce (Tabela 2), com o melhor ajuste em novembro (Figura 3). As piores estimativas do modelo, que considera o índice $\mathrm{Z}$ como variável independente, são para as mesorregiões Triângulo/ Alto Paranaíba e Central Mineira, exatamente as que tiveram as maiores quedas na produtividade em função da limitação por água (Figura 3). Em quase todas as localidades estudadas, o modelo do índice $\mathrm{Z}$ tende a superestimar a produtividade estimada pela metodologia AEZ nos anos agrícolas com deficiência hídrica, ou nas safras com menor produtividade, e subestimar os anos agrícolas com maior produtividade (Figura 3 ).

Os melhores ajustes entre a produtividade estimada pelo índice $\mathrm{Z}$ e a produtividade estimada pela metodologia AEZ foram obtidos nas mesorregiões Vale do Rio Doce, Sul e Sudoeste, com valores de RMSE e MAE iguais a 418,46 e $594,88 \mathrm{~kg} / \mathrm{ha}$ e 336,43 e 444,49 kg/ha, respectivamente (Tabela 2). Nessas mesorregiões, também se obteve bons valores de coeficiente de determinação $\left(\mathrm{r}^{2}\right)$ e índice de concordância de Willmott (d), com valores de $r^{2}$ iguais a 0,69 e 0,49 e valores de d iguais a 0,90 e 0,80 , respectivamente. As mesorregiões Jequitinhonha e Zona da Mata apresentaram os valores mais baixos de $\mathrm{r}^{2}(0,12$ e 0,16$)$ e dw índice de concordância de Willmott (0,48 e 0,52, respectivamente). Contudo, os resultados encontrados nesse estudo estão de acordo com a literatura, como Quiring e Papakryiakou (2003), que encontraram baixos valores para o coeficiente de determinação (variando de 0,15 a 0,47 ); e os índices de concordância entre 0,14 e 0,76 , na avaliação do desempenho do índice de seca mais apropriado ao monitoramento da seca agrícola no cultivo do trigo nas pradarias canadenses; e como Gois (2005), que avaliando o desempenho do método dos Decis para produção de milho nas em Minas Gerais obteve valores de $\mathrm{r}^{2}$ de 0,$08 ; 0,03$ e 0,001, para as mesorregiões Zona da Mata, Triângulo/Alto Paranaíba e Metropolitana de Belo Horizonte, respectivamente, e índice de concordância de 0,2228 para a mesorregião Triângulo/Alto Paranaíba e de 0,0443 para a mesorregião Metropolitana de Belo Horizonte.

O desempenho do índice Z, para estimar as variações na produtividade estimada pela metodologia $\mathrm{AEZ}$, foi satisfatório, divergindo apenas nas variações da produtividade estimada pela metodologia AEZ nos anos agrícolas de 2008 e 2009 (Figura 4). Contudo, essa divergência não necessariamente implica em restrições do índice $Z$, pois, considerando que os valores de produtividade estimados pela metodologia AEZ foram limitados somente por água, de maneira geral, a produtividade 

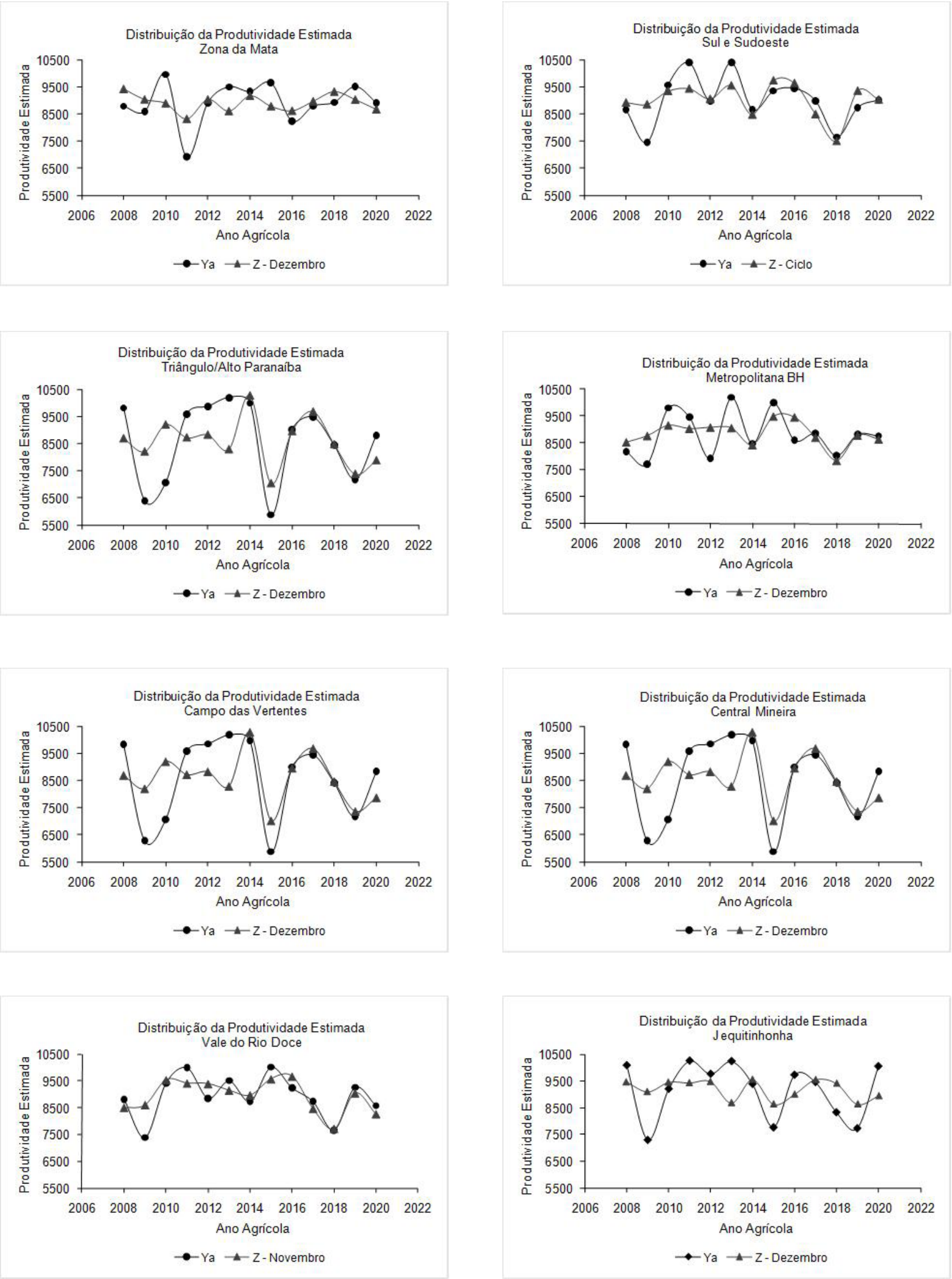

Figura 3 - Distribuição da produtividade estimada pela metodologia da zona agroecológica (AEZ) e pelo índice Z para as mesorregiões de Minas Gerais, considerando o cenário A1B do modelo ECHAM5/MPI-OM, no período de 2008 a 2020 
Tabela 2 - Desempenho do índice Z para as mesorregiões de Minas Gerais

\begin{tabular}{l|c|c|c|c|c}
\hline Mesorregiões & RMSE & MAE & d & $\mathbf{r}^{2}$ & Período \\
\hline Zona da Mata & 674,68 & 529,87 & 0,52 & 0,16 & Dezembro \\
Sul Sudoeste & 594,88 & 444,49 & 0,80 & 0,49 & Ciclo* \\
Triângulo/Alto Paranaíba & 1138,86 & 902,15 & 0,72 & 0,36 & Dezembro \\
Metropolitana Belo Horizonte & 641,26 & 515,63 & 0,67 & 0,32 & Dezembro \\
Campo das Vertentes & 643,54 & 548,60 & 0,71 & 0,37 & Dezembro \\
Central Mineira & 1154,77 & 914,50 & 0,71 & 0,35 & Dezembro \\
Jequitinhonha & 934,31 & 788,81 & 0,48 & 0,12 & Dezembro \\
Vale do Rio Doce & 418,46 & 336,43 & 0,90 & 0,69 & Novembro \\
\hline
\end{tabular}

*Ciclo = Outubro, Novembro, Dezembro e Janeiro.

gerada pelo modelo do índice $\mathrm{Z}$ apresentou boa concordância com a produtividade calculada pela metodologia AEZ (Tabela 2, Figura 3).

\section{CONCLUSÕES}

Este estudo mostrou que, se as cultivares, práticas de manejo, e as tecnologias atuais forem mantidas, e caso as condições futuras do clima tenderem ao cenário de emissões A1B, a mudança projetada do clima terá um efeito negativo na produtividade de milho nas mesorregiões analisadas, quando se analisa apenas a água como fator de limitação para a produtividade. Porém, sabe-se que existe uma grande incerteza em relação aos valores de precipitação modelados para o clima futuro. Além disso, a influência do clima nas culturas se dá por meio de uma combinação dos diferentes elementos climáticos. Portanto, é necessário um estudo elaborado, que analise todas essas interações climáticas, bem como, práticas de manejo, influências do solo, entre outras.

$\mathrm{O}$ índice $\mathrm{Z}$ foi satisfatório para estimar as variações na produtividade estimada pela metodologia AEZ, podendo ser utilizado com uma ferramenta de análise para a relação seca/ produtividade. Porém, cabe ressaltar que todo o estudo foi realizado com projeções de dados climáticos futuros. Sendo assim, a aplicação desse estudo pode conter tendências do modelo e do cenário utilizado.

\section{AGRADECIMENTOS}

Ao Conselho Nacional de Desenvolvimento Científico e Tecnológico (CNPq) pelo apoio financeiro.

\section{REFERÊNCIAS BIBLIOGRÁFICAS}

BARRIOS, S.; OUATTARA, B.; STROBL, E. The impact of climatic change on agricultural production: Is it different for Africa? Food Policy, v. 33, p. 287-370, 2008.
BURKE, E. J.; BROWN, S. J.; CHRISTIDIS, N. Modeling the recent evolution of global drought and projections for the twenty-first century with the Hadley Centre Climate Model. Journal of Hidrometeorology, v.7, p.1113-1125, 2006.

CALLINOR, A. J.; WHEELER, T. R.; CRAUFURD, P. Q., FERRO, C. A. T.; STEPHENSON, D. B. Adaptation of crops to climate change through genotypic responses to mean and extreme temperatures. Agriculture Ecosystems \& Environment, v.119, p.190-204, 2007.

DENG, X.; HUANG, J.; ROZELLE, S.; UCHIDA, E. Cultivated land conversion and potential agricultural productivity in China. Land Use Policy, v.23, p.372-384, 2006.

DOORENBOS, J.; KASSAM,A. H. Yield response to water. FAO: Irrigation and Drainage Paper, 33, Rome, FAO, 172 p. 1979.

EWERT, F.; ROUNSEVELL, M. D. A.; REGINSTER, I.; METZGER, M. J.; LEEMANS, R. Future scenarios of European agricultural land use. I. Estimating changes in crop productivity. Agriculture, Ecosystems and Environment, v.107, p.101-116, 2005.

FAO, 1978-81. Report on the agro-ecological zones project. World Soil Resources Report 48, FAO, Rome, 1978.

FISCHER, G.; VELTHUIZEN, H. V.; NACHTERGAELE, F. O. Global agroecological zones assessment: methodology and results. Interim report. IIASA, Laxenburg, Austria, 2000.

FISCHER, G.; VELTHUIZEN, H. V.; SHAH, M.; NACHTERGAELE, F. O. Global agro-ecological assessment for agriculture in the 21 st century: Methodology and results. IIASA RR-02-02, IIASA, Laxenburg, 2002.

GOIS, G. de. Caracterização da seca e seus efeitos na produção da cultura do milho para diferentes regiões do Estado de Minas Gerais. 2005. 116 f. Dissertação (Mestrado em Meteorologia Agrícola) - Programa de Pós-Graduação em Meteorologia Agrícola - Universidade Federal de Viçosa, Viçosa, 2005.

HEIM JUNIOR, R. R. A review of twentieth - century drought indices used in the United States. Bulletin of the American Meteorological Society, v.83, p.1149-1165, 2002. 
HUGHES, M. K.; DIAZ, H. F. Climate variability and change in the drylands of Western North America. Global and Planetary Chang, v.68, p.111-118, 2008.

IPCC - Intergovernmental Panel on Climate Change: Climate Change 2007: The physical science basis. Cambridge, 18 p. 2007.

KARL, T. The sensitivity of the Palmer drought severity index and Palmer's Z-index to their calibration coefficients including potential evapotranspiration. Journal of Climate and Applied Meteorology, v.25, p.77-86, 1986.

KASSAM, A. H. Net biomass production and yield of crops, FAO, Rome, 1977.

KEYANTASH, J.; DRACUP, J. A. The quantification of drought: An evaluation of drought indices. Bulletin of the American Meteorological Society, v.83, p.1167-1180, 2002.

LEAL, B. G. Sistema computacional para a avaliação e estimativa das produtividades potencial e real de culturas agrícolas. 2000. 97p. Tese (Doutorado em Engenharia Agrícola) - Programa de Pós-Graduação em Engenharia Agrícola - Universidade Federal de Viçosa, Viçosa, 2000.

LOBELL, D. B. Change in diurnal temperature range and national cereal yields. Agricultural and Forest Meteorology, v.145, p.229-238, 2007.

LUO, Q.; BELLOTI, W.; WILLIAMS, M.; BRYAN, B. Potential impact of climate change on wheat yield in South Australia. Agricultural and Forest Meteorology, v.132, p.273-285, 2005.

MARSLAND, S .J.; HAAK, H.; JUNGCLAUS, J. H.; LATIF, M.; RÖSKE, F. The Max Planck Institute global ocean/seaice model with orthogonal curvilinear coordinates. Ocean Modell, v.5, p.91-127, 2003.

OLIVEIRA, L. J. C. Mudanças climáticas e seus impactos nas produtividades das culturas do feijão e do milho no Estado de Minas Gerais. 2007. 67 f. Dissertação (Mestrado em Meteorologia Agrícola) - Programa de Pós-Graduação em Meteorologia Agrícola - Universidade Federal de Viçosa, Viçosa, 2007.
PALMER, W. C. Meteorological drought. Research Paper n. 45, Weather Bureau, Washington, 58 p., 1965.

PINTO, H. S.; ASSAD, E. D.; ZULLO JR, J.; AVILA, A. M. H. de . Variabilidade Climática. In: Água, Agricultura e Meio Ambiente Avanços e Desafios. Jaguariuna, SP. Embrapa Meio Ambiente, v. 1. p. 1-13, 2003.

QUIRING, S. M.; PAPAKRYAIKOU, T. N. An evaluation of agricultural drought indices for the Canadian prairies. Agricultural and Forest Meteorology, v.118, p.49-62, 2003.

RICHTER, G. M.; SEMENOV, M. A. Modelling impacts of climate change on wheat yields in England and Wales: assessing drought risks. Agricultural Systems, v.84, p.7797, 2005.

ROECKNER, E.; BÄUML, G.; BONAVENTURA, L.; BROKOPF, R.; ESCH, M.; GIORGETTA, M.; HAGEMANN, S.; KIRCHNER, I.; KORNBLUEH, L.; MANZINI, E.; RHODIN, A.; SCHLESE, U.; SCHULZWEIDA, U.; TOMPKINSAND, A. The atmospheric general circulation model ECHAM5, Part I: Model description. Max-PlanckInstitut for Meteorology, Rep. 349, 127 p., 2003.

SILVA JUNIOR, J. L. C. Simulação da produtividade das culturas do milho e do feijão, baseada nos cenários de mudanças climáticas globais, do estado de Minas Gerais. 2007. Dissertação (Doutorado em Meteorologia Agrícola) - Programa de Pós-Graduação em Meteorologia Agrícola - Universidade Federal de Viçosa, Viçosa, 2007.

ZHANG, X. C.; LIU, W. Z. Simulating potential response of hydrology, soil erosion, and crop productivity to climate change in Changwu tableland region on the Loess Plateau of China. Agricultural and Forest Meteorology, Amsterdam, v. 131, p.127-142, 2005.

WILLMOTT, C. J. Some comments on the evaluation of model performance. Bulletin of the American Meteorological Society, Lancaster, v.63, n.11, p.1309-1313, 1982. 